\title{
Vacuum Decay on a Brane World
}

\author{
Stephen C. Davis and Sylvain Bréchet \\ Institute of Theoretical Physics, École Polytechnique Fédérale de Lausanne, CH-1015 Lausanne, Switzerland
}

\begin{abstract}
The bubble nucleation rate for a first order phase transition occurring on a brane world is calculated. Both the Coleman-de Luccia thin wall instanton and the Hawking-Moss instanton are considered. The results are compared with the corresponding nucleation rates for standard fourdimensional gravity.
\end{abstract}

\section{INTRODUCTION}

It is widely believed that as the universe cools it undergoes a series of phase transitions. Each of these involves a transition from a metastable "false vacuum" ground state to a stable one, the "true vacuum". In the case of a first order transition, this change is initiated by the nucleation of bubbles of true vacuum. These then expand at a speed asymptotically approaching that of light, converting false vacuum into true as they grow. The decay of false vacuum was first studied by Kobzarev, Okun and Voloshin [1]. It was shown by Coleman 2] that the nucleation rate could be calculated using instantons. This was extended by Coleman and de Luccia, who showed that the rate at which the decay occurs is altered by gravity 3. This will be most significant in the early universe when the curvature is high. Most previous work on the effects of gravity on vacuum decay has assumed, not surprisingly, that gravity is described by general relativity. However this may not be true, in which case the phase transition, and any related processes such as defect formation, will be altered.

There has been a lot of interest in the string-motivated brane world scenario, in which our universe is a 3-brane embedded in a higher dimensional "bulk" space-time. The extra dimensional effects produce modifications to gravity. The particular brane model that we will consider is the Randall-Sundrum II model [4], which has a single extra dimension and a warped bulk spacetime. The warping allows conventional gravity to be obtained on the brane in the small curvature limit. However in more extreme circumstances, such as during the early universe [5], gravity will behave differently.

In this article we calculate the vacuum decay rate on a brane world. In section I we extend the work of Coleman and de Luccia (CdL) to the Randall-Sundrum model. Analytic determination of the correct instanton for this method is not possible, and so we will instead use some approximate solutions. In section III we look at the CdL thin wall instanton, and consider some limiting cases of it. In section IV we find the decay rate for the HawkingMoss instanton [6]. Although we are unable to provide a completely general analysis, our results do allow vacuum decay in the brane world to be compared with the results for standard gravity.

\section{BOUNCE ACTION}

We will consider a toy model with one scalar field, $\phi$, whose potential is $U(\phi)$. The potential has minimums at $\phi_{\mathrm{F}}$ and $\phi_{\mathrm{T}}$, and $U_{\mathrm{F}} \equiv U\left(\phi_{\mathrm{F}}\right)$ is greater than $U_{\mathrm{T}} \equiv U\left(\phi_{\mathrm{T}}\right)$. Thus $\phi=\phi_{\mathrm{F}}$ is a metastable false vacuum state, and $\phi=\phi_{\mathrm{T}}$ is the global minimum of the potential and hence the true vacuum state. In this article we will take $U_{\mathrm{F}}$ and $U_{\mathrm{T}}$ to be both positive.

In the semi-classical limit, the bubble nucleation rate per unit volume is given by [2]

$$
\Gamma / V=A e^{-B / \hbar}[1+O(\hbar)]
$$

The bounce action, $B$, is defined as

$$
B=S_{E}(\phi)-S_{E}\left(\phi_{\mathrm{F}}\right)
$$

where $S_{E}$ is the Euclidean action, defined as minus the analytic continuation of the usual action to imaginary time. The instanton $\phi$, called the "bounce", is the solution of the field equations which minimises $B$ and which traverses the potential barrier between the true and false vacuums. In this article we will ignore the factor $A$, and just determine $B$, since it gives the dominant contribution to the behaviour of $\Gamma / V$.

For a brane world, with a $Z_{2}$ symmetric bulk, the Euclidean action is given by $S_{E}=S_{E}^{(B)}+S_{E}^{(b)}$, where the bulk and brane contributions are respectively

$$
\begin{array}{r}
S_{E}^{(B)}=\frac{1}{2 \kappa_{5}^{2}} \int d^{5} x \sqrt{g}\left(-R^{(5)}+2 \Lambda_{5}\right), \\
S_{E}^{(b)}=\frac{1}{\kappa_{5}^{2}} \int d^{4} x \sqrt{h}\left(2 K+\lambda_{4}\right) \\
+\int d^{4} x \sqrt{h}\left(\frac{1}{2}(D \phi)^{2}+U(\phi)\right) .
\end{array}
$$

The induced metric metric on the brane is $h_{a b}=g_{a b}-$ $n_{a} n_{b}$, where $n_{a}$ is the (outward) brane normal. $K=$ $h^{a b} K_{a b}$ is the Gibbons-Hawking boundary term [7], with $K_{a b}=h_{a}^{c} \nabla_{c} n_{b}$ being the extrinsic curvature. Although we have a five dimensional gravity theory, the scalar field is restricted to the brane. The induced derivative is defined as $D_{a} \phi=h_{a}^{b} \nabla_{b} \phi$.

As with the Randall-Sundrum model, we take $\Lambda_{5}=$ $-6 / \ell^{2}$ and $\lambda_{4}=6 / \ell$. We could have absorbed $\lambda_{4}$ into $U$, although by keeping them separate we can more 
easily compare our results with those of standard fourdimensional gravity. The effective four dimensional gravitational coupling on the brane is given by $\kappa^{2}=\kappa_{5}^{2} / \ell$.

Variation of the action gives the field equations

$$
\begin{gathered}
G_{a b}^{(5)}+\Lambda_{5} g_{a b}=0 \\
-2\left(K_{a b}-K h_{a b}\right)+\lambda_{4} h_{a b} \\
=\kappa_{5}^{2}\left(D_{a} \phi D_{b} \phi-\frac{1}{2} h_{a b}(D \phi)^{2}-h_{a b} U(\phi)\right) \\
D^{2} \phi=\frac{d U}{d \phi} .
\end{gathered}
$$

In flat space the bounce is $O(4)$ symmetric [8]. This is usually assumed when Einstein gravity is included in the theory, and we will also assume it for the brane world. This implies

$$
d s_{\text {brane }}^{2}=d \xi^{2}+a(\xi)^{2} d \Omega_{3}^{2}, \quad \phi=\phi(\xi),
$$

where $d \Omega_{3}^{2}$ is the metric of $S^{3}$. For the solution to have a finite action, we require $a(0)=a\left(\xi_{\max }\right)=0$ and $\dot{\phi}(0)=$ $\dot{\phi}\left(\xi_{\max }\right)=0$.

The general $O(4)$ symmetric solution of the field equations (5 7) can be obtained be analytically continuing a brane world cosmology solution. Using the results of Ida [9], we obtain

$$
d s_{\mathrm{Bulk}}^{2}=f(r) d \Xi^{2}+\frac{d r^{2}}{f(r)}+r^{2} d \Omega_{3}^{2},
$$

where $f(r)=1+r^{2} / \ell^{2}-\mathcal{C} / r^{2}$. The parameter $\mathcal{C}$ corresponds to the black hole mass in the original cosmological solution. A brane-based observer would perceive it as "dark radiation". The position of the brane is given by $r=a(\xi), \Xi=\Xi_{b}(\xi)$. In each half of the $Z_{2}$ symmetric bulk, $r$ ranges from 0 to $a$.

The brane field equations (6) and (7) reduce to

$$
\begin{gathered}
\frac{\dot{a}^{2}}{a^{2}}=\frac{f(a)}{a^{2}}-\left\{\frac{\kappa_{5}^{2}}{6}\left(U-\frac{\dot{\phi}^{2}}{2}\right)+\frac{1}{\ell}\right\}^{2}, \\
\ddot{\phi}+\frac{3 \dot{a}}{a} \dot{\phi}-\frac{d U(\phi)}{d \phi}=0 .
\end{gathered}
$$

The fact that the two metrics agree at $r=a$, gives us the relation

$$
\frac{d \Xi}{d \xi}=\frac{\sqrt{f(a)-\dot{a}^{2}}}{f(a)}
$$

on the brane.

With the aid of the field equation (5), the bulk part of the action simplifies to

$$
S_{E}^{(B)}=-\frac{8 \pi^{2}}{3 \kappa_{5}^{2}} \Lambda_{5} \int_{r=0}^{r=a} r^{3} d r d \Xi
$$

Integrating with respect to $r$, and using the relation (12), we obtain

$$
S_{E}^{(B)}=\frac{2 \pi^{2}}{3 \ell^{2}} \int d \xi \frac{a^{5}}{f(a)}\left(U-\frac{\dot{\phi}^{2}}{2}+\frac{6}{\kappa_{5}^{2} \ell}\right) .
$$

Using the trace of the junction condition (6), the brane part of the action reduces to

$$
S_{E}^{(b)}=-\frac{2 \pi^{2}}{3} \int d \xi a^{3}\left(U-\frac{\dot{\phi}^{2}}{2}+\frac{6}{\kappa_{5}^{2} \ell}\right) .
$$

Before we try to evaluate the expression for $B=$ $S_{E}(\phi)-S_{E}\left(\phi_{\mathrm{F}}\right)$, it is convenient to add a total derivative of the form $-\left(2 / \kappa^{2}\right) \partial_{\xi}\left(a \dot{a}^{2}\right)$ to the action. This will not alter the value of $B$, although it does make the thin wall approximation (see next section) easier to apply. Adding together all the above contributions to the action, we obtain

$$
\begin{aligned}
S_{E}(\phi) & =2 \pi^{2} \int d \xi a^{3}\{2 U \\
& +\frac{1}{3}\left(U-\frac{\dot{\phi}^{2}}{2}\right)\left[\frac{a^{2}}{\ell^{2} f(a)}-1+\frac{\kappa_{5}^{2} \ell}{2}\left(\frac{\dot{\phi}^{2}}{2}+U\right)\right] \\
& \left.+\frac{2}{\kappa_{5}^{2} \ell}\left(\frac{a^{2}}{\ell^{2} f(a)}-\frac{\ell^{2}}{a^{2}}[f(a)+1]\right)\right\} .
\end{aligned}
$$

\section{THIN WALL APPROXIMATION}

The expression for $B=S_{E}(\phi)-S_{E}\left(\phi_{\mathrm{F}}\right)$ is too complicated to evaluate analytically, so we will instead use the thin wall approximation. This consists of taking the bounce instanton to be a ball of true vacuum surrounded by false vacuum, with a thin wall at $a=\bar{a}$ separating the two regions. Away from the wall $\phi$ is constant and so the action simplifies considerably. The thin wall approximation holds when the wall thickness is far smaller than $\bar{a}$, which will be the case when the energy difference $\epsilon=U_{\mathrm{F}}-U_{\mathrm{T}}$ is small compared to the barrier height.

On the wall $a$ is roughly constant, and so in the scalar field equation (11) the second term can be dropped. We also approximate the potential on the wall by $U_{0}(\phi)=$ $U(\phi)+O(\epsilon)$. The approximate potential $U_{0}$ also has minimums at $\phi_{\mathrm{T}}$ and $\phi_{\mathrm{F}}$, but with $U_{0}\left(\phi_{\mathrm{F}}\right)=U_{0}\left(\phi_{\mathrm{T}}\right)$. To leading order in $\epsilon$, we can approximate the equation for $\phi$ on the wall by $\ddot{\phi}=d U_{0} / d \phi$. This is solved by

$$
\frac{1}{2}(\dot{\phi})^{2}-U_{0}(\phi)=-U_{0}\left(\phi_{\mathrm{F}}\right),
$$

which allows the leading order contribution to $B$ from the wall to be obtained.

We will now use the thin wall approximation for the brane action (16), and evaluate the parameter $B$. For simplicity we will assume there is no black hole in the 
bulk space, so $\mathcal{C}=0$. For the space outside the wall we have $B_{\text {outside }}=S_{E}\left(\phi_{\mathrm{F}}\right)-S_{E}\left(\phi_{\mathrm{F}}\right)=0$. On the wall we use the expression (17) and $a=\bar{a}$, to obtain

$$
B_{\mathrm{wall}}=S_{E}(\phi)-S_{E}\left(\phi_{\mathrm{F}}\right)=2 \pi^{2} \bar{a}^{3} \alpha_{0} S_{1},
$$

where $\alpha_{0}=\left(1+\kappa_{5}^{2} \ell U_{0}\left(\phi_{\mathrm{F}}\right) / 6\right)$ and

$$
S_{1}=\int_{\phi_{\mathrm{T}}}^{\phi_{\mathrm{F}}} d \phi\left\{2\left[U_{0}(\phi)-U_{0}\left(\phi_{\mathrm{F}}\right)\right]\right\}^{1 / 2} .
$$

Before evaluating the contribution to $B$ from inside the wall, we will define some notation

$$
\begin{gathered}
\alpha_{i}=1+\frac{\kappa_{5}^{2} \ell U_{i}}{6}, \\
H_{i}^{2}=\frac{\kappa_{5}^{4}}{36} U_{i}^{2}+\frac{\kappa_{5}^{2}}{3 \ell} U_{i}=\frac{\alpha_{i}^{2}-1}{\ell^{2}} .
\end{gathered}
$$

with $i$ being ' $\mathrm{T}$ ' or ' $\mathrm{F}$ '.

Inside the wall, $\phi$ is a constant. The Friedmann equation (10) implies

$$
d \xi= \pm d a\left(1-H_{\mathrm{T}}^{2} a^{2}\right)^{-1 / 2}
$$

The choice of sign depends which half of the four dimensional spacetime we are in. The positive sign applies when $\xi$ is near 0 , and the negative sign when it is near $\xi_{\max }$. We see that the above coordinate change is not one to one and that, for a given $\bar{a}$, there are two possible CdL instantons.

If the upper sign in eq. (22) applies for the entire region inside the wall then, after changing variables, we obtain $B_{\text {inside }}=\mathcal{S}\left(\phi_{\mathrm{T}}, \bar{a}\right)-\mathcal{S}\left(\phi_{\mathrm{F}}, \bar{a}\right)$, where

$$
\begin{aligned}
\mathcal{S}\left(\phi_{i}, b\right)= & \frac{4 \pi^{2}}{\kappa^{2}} \int_{0}^{b} \frac{a d a}{\left(1-H_{i}^{2} a^{2}\right)^{1 / 2}} \\
& \times\left\{1-\frac{a^{2}}{\ell^{2}+a^{2}} \alpha_{i}-3\left(1-H_{i}^{2} a^{2}\right)\right\},
\end{aligned}
$$

i.e. the contribution to $S_{E}$ (for constant $\phi$ ) from the region $0<a<b$. The above expression evaluates to

$$
\begin{aligned}
& \mathcal{S}\left(\phi_{i}, a\right)=\frac{4 \pi^{2}}{\kappa^{2}}\left\{\frac{\ell^{2}}{2} \ln \frac{\left(\sqrt{1-H_{i}^{2} a^{2}}-\alpha_{i}\right)\left(1+\alpha_{i}\right)}{\left(\sqrt{1-H_{i}^{2} a^{2}}+\alpha_{i}\right)\left(1-\alpha_{i}\right)}\right. \\
& \left.+\frac{\ell^{2}}{\alpha_{i}+1}\left(1-H_{i}^{2} a^{2}\right)^{1 / 2}+\frac{1}{H_{i}^{2}}\left(1-H_{i}^{2} a^{2}\right)^{3 / 2}-\frac{\alpha_{i}}{H_{i}^{2}}\right\}
\end{aligned}
$$

which will give $B_{\text {inside }}$.

It is also possible to have a thin wall instanton which has different signs in the relation (22) on different sides of the wall. In this case the part of $S_{E}\left(\phi_{\mathrm{F}}\right)$ inside the wall will have contributions from both halves of the spacetime, and we find

$$
B_{\text {inside }}=\mathcal{S}\left(\phi_{\mathrm{T}}, \bar{a}\right)-2 \mathcal{S}\left(\phi_{\mathrm{F}}, 1 / H_{\mathrm{F}}\right)+\mathcal{S}\left(\phi_{\mathrm{F}}, \bar{a}\right)
$$

with $1 / H_{\mathrm{F}}$ being the maximum value of $a$ for $\phi \equiv \phi_{\mathrm{F}}$.

The expression (1) for $\Gamma / V$ is evaluated for the instanton which minimises the action $B$. We can estimate this by minimising the our approximate expression for $B$ with respect to $\bar{a}$. Using the above expressions, we find that this is the case when

$$
\begin{gathered}
\frac{3}{2} \kappa^{2} \bar{a} S_{1} \alpha_{0}+\left\{\left[1-\frac{\bar{a}^{2}}{\ell^{2}+\bar{a}^{2}} \alpha_{\mathrm{T}}\right]\left(1-H_{\mathrm{T}}^{2} \bar{a}^{2}\right)^{-1 / 2}\right. \\
\left.-3\left(1-H_{\mathrm{T}}^{2} \bar{a}^{2}\right)^{1 / 2}\right\}-\sigma\left\{\mathrm{T} \leftrightarrow{ }_{\mathrm{F}}\right\}=0
\end{gathered}
$$

The sign $\sigma$ is equal to -1 if the expression (25) is applicable, and 1 otherwise.

At late times brane cosmology will reduce to standard four dimensional cosmology. This occurs when $\ell$ is small relative to other length scales in the theory. Similarly we expect to obtain the usual four dimensional tunnelling rate when $\ell$ is small. Taking $\ell \ll 6 /\left(\kappa_{5}^{2} U\right)$ and $\ell \ll \bar{a}$, the equations for $B$ and $\bar{a}$ reduce to the conventional expressions, as obtained by Parke [10].

Even with the simplifications resulting from the thin wall approximation, eq. (26) is still algebraically complicated. Rather than trying to solve it analytically, it is more instructive to look at various limiting cases. Gravitational effects will be most significant when the vacuum energy or the barrier size is large, and so we will concentrate on these limiting cases.

\section{(i) Large Vacuum Energy Limit}

The expression for $\bar{a}$ will simplify if we suppose that $U_{\mathrm{F}}, U_{\mathrm{T}} \gg \epsilon$. In this case $\bar{a}$ will be close to its maximal value, and so $\bar{a}^{2}=(1-\eta) / H_{\mathrm{F}}^{2}$ with $\epsilon / U \ll \eta \ll 1$. We take $\sigma=1$ in eq. (26).

If $\kappa_{5}^{2} \ell U_{\mathrm{F}} \ll \eta$, then higher dimensional contributions to gravity will be small. In this case we obtain the same results as for standard four-dimensional gravity. These are $\eta \sim \epsilon^{2} /\left(\kappa^{2} U S_{1}^{2}\right)$ and

$$
B_{4 D}=\frac{6 \sqrt{3} \pi^{2} S_{1}}{\kappa^{3} U^{3 / 2}}
$$

The limits used will be self consistent if $\epsilon / U \ll S_{1}^{2} \kappa^{2} / \epsilon \ll$ 1 and $\ell \ll \epsilon /\left(S_{1} \kappa^{2} U\right)$. This case is contained within the limit (i) in ref. 10]. For comparison, the bounce action in the absence of gravity is $B_{0}=27 \pi^{2} S_{1}^{4} /\left(2 \epsilon^{3}\right)$, and so we see that the effects of gravitation decrease $B$.

For higher vacuum energies brane gravity effects will be important. They will be most significant when $\kappa_{5}^{2} \ell U \gg$ 1. In this limiting brane gravity case $\eta^{3 / 2} \sim \epsilon /\left(\kappa_{5}^{2} U S_{1}\right)$ and

$$
B_{B W}=\frac{72 \pi^{2} \ell S_{1}}{\kappa^{3} \kappa_{5} \ell^{1 / 2} U^{2}} \ll B_{4 D} .
$$

Consistency requires $\epsilon /\left[\kappa_{5}^{2} \ell U^{2}\right] \ll S_{1}^{2} \kappa^{2} / \epsilon \ll U /\left[\kappa_{5}^{2} \ell \epsilon^{2}\right]$. 
For both the above expressions, it is the contribution from the wall that dominates $B$. The inclusion of gravitation in the theory reduces $B$, and so increases the nucleation rate. If we use brane gravity instead of conventional gravity, the nucleation rate is increased even further, especially when $U$ is greater than the brane tension, $\lambda_{4} / \kappa_{5}^{2}$.

\section{(ii) Large Barrier Limit}

We can also obtain approximate analytic expressions for $\bar{a}$ and $B$ when $H_{\mathrm{F}} \bar{a} \ll 1$ and $\sigma=-1$. This implies that the bounce instanton is close to the maximum size that will fit in the spacetime. This case corresponds to the limit (ii) considered in ref. [10].

For $\kappa_{5}^{2} \ell U_{\mathrm{F}} \ll 1$ we re-obtain the result for conventional gravity. This has $\bar{a} \sim 1 /\left(\kappa^{2} S_{1}^{2}\right)$ and, using eq. (25),

$$
B_{4 D}=\frac{24 \pi^{2}}{\kappa^{4} U_{\mathrm{F}}}
$$

This limit is valid when $\kappa^{2} S_{1}^{2} \gg U_{\mathrm{F}}$, in other words when the barrier height is large compared to the vacuum energy.

Brane effects will be most significant when $\kappa_{5}^{2} \ell U_{\mathrm{F}} \gg 1$. In this case we find $\bar{a} \sim \kappa_{5}^{2} \ell S_{1}$ and

$$
B_{B W}=\frac{1152 \pi^{2}}{\kappa^{4} \kappa_{5}^{4} \ell^{2} U_{\mathrm{F}}^{3}} \ll B_{4 D}
$$

which is valid when $\kappa_{5}^{4} S_{1}^{2} \gg 1 /\left(\kappa_{5}^{2} \ell U_{\mathrm{F}}\right)$.

For this limiting case the dominant contribution to $B$ comes from the region inside the wall. Again we wee that gravity increases the nucleation rate, and brane gravity increases it even more.

\section{HAWKING-MOSS INSTANTON}

As well as the approximate CdL instanton, we will also consider the Hawking-Moss instanton. For this $\phi$ is a constant, and sits at the top of the potential barrier, which we denote by $\phi=\phi_{\mathrm{M}}$. If $U(\phi)$ is very large, it is expected that the Hawking-Moss instanton will provide a better approximation of bounce than the thin wall $\mathrm{CdL}$ instanton. The bounce action is $B=2 \mathcal{S}\left(\phi_{\mathrm{M}}, 1 / H_{\mathrm{M}}\right)-$ $2 \mathcal{S}\left(\phi_{\mathrm{F}}, 1 / H_{\mathrm{F}}\right)$, which evaluates to

$$
B=\frac{4 \pi^{2}}{\kappa^{2}}\left(\frac{\ell^{2}}{2} \ln \frac{\left(\alpha_{\mathrm{M}}+1\right)\left(\alpha_{\mathrm{F}}-1\right)}{\left(\alpha_{\mathrm{M}}-1\right)\left(\alpha_{\mathrm{F}}+1\right)}-\frac{\alpha_{\mathrm{M}}}{H_{\mathrm{M}}^{2}}+\frac{\alpha_{\mathrm{F}}}{H_{\mathrm{F}}^{2}}\right) .
$$

For small $\kappa_{5}^{2} \ell U$ this reduces to the standard result

$$
B_{4 D}=\frac{24 \pi^{2}}{\kappa^{4}}\left(\frac{1}{U_{\mathrm{F}}}-\frac{1}{U_{\mathrm{M}}}\right)
$$

On the other hand if $\kappa_{5}^{2} \ell U \gg 1$, brane effects will dominate and

$$
B_{B W}=\frac{1152 \pi^{2}}{\kappa^{4} \kappa_{5}^{4} \ell^{2}}\left(\frac{1}{U_{\mathrm{F}}^{3}}-\frac{1}{U_{\mathrm{M}}^{3}}\right) \ll B_{4 D} .
$$

As with CdL instanton, we see that brane effects significantly reduce $B$, and hence increase the nucleation rate.

\section{CONCLUSION}

We have evaluated the bubble nucleation rate for first order phase transitions on a Randall-Sundrum brane world. If the potential is smaller than the brane tension, we obtain (to leading order) the standard four dimensional results. This is not surprising, since for small curvature, brane gravity reduces to standard four dimensional general relativity. On the other hand if the potential is larger than the brane tension, the non-standard brane gravity effects will be significant. In all the cases that we considered, the nucleation rate was significantly increased by brane gravity. This suggests that, at least for positive definite potentials, the nucleation rate in brane models will be higher than for conventional gravity. This is analogous to the situation in brane cosmology, where the Hubble parameter is larger than in the equivalent conventional cosmology.

To fully model a phase transition, we also need to consider the expansion of the bubbles after nucleation. In other work it has been shown that for brane models a higher nucleation rate is required for the transitions to complete successfully [1]. Our results suggest this problem could be fixed by the brane gravity. However it should be noted that in the early universe finite temperature effects [12] will be significant, and our expressions are for $T=0$. As well as corrections to $B$, the factor $A$ in eq. (1) also needs to be determined. On dimensional grounds this is usually taken to be of order $T^{4}$. However for $\kappa_{5}^{2} \ell T^{4} \gg 1$ brane gravity effects will be significant and we would expect $A$ to be some combination of $T^{4}$ and $\kappa_{5}^{2} \ell$ instead. If this is the case the analysis of ref. [1] (which assumes $A \sim T^{4}$ ) will also be altered.
[1] I. Y. Kobzarev, L. B. Okun and M. B. Voloshin, "Bubbles In Metastable Vacuum," Sov. J. Nucl. Phys. 20 (1975) 644 [Yad. Fiz. 20 (1974) 1229].

[2] S. Coleman, "Fate of the false vacuum: Semiclassical the- ory," Phys. Rev. D 15 (1977) 2929;

C. G. Callan and S. Coleman, "Fate of false vacuum. II. First quantum corrections," Phys. Rev. D 16 (1977) 1762 . 
[3] S. Coleman and F. De Luccia, "Gravitational effects on and of vacuum decay," Phys. Rev. B 21 (1980) 3305.

[4] L. Randall and R. Sundrum, "An alternative to compactification," Phys. Rev. Lett. 83, 4690 (1999) hep-th/9906064.

[5] P. Binetruy, C. Deffayet, U. Ellwanger and D. Langlois, "Brane cosmological evolution in a bulk with cosmological constant," Phys. Lett. B 477 (2000) 285 hep-th/9910219.

[6] S. W. Hawking and I. G. Moss, "Supercooled Phase Transitions In The Very Early Universe," Phys. Lett. B 110 (1982) 35.

[7] G. W. Gibbons and S. W. Hawking, "Action Integrals And Partition Functions In Quantum Gravity," Phys. Rev. D 15 (1977) 2752.
[8] S. R. Coleman, V. Glaser and A. Martin, "Action Minima Among Solutions To A Class Of Euclidean Scalar Field Equations," Commun. Math. Phys. 58 (1978) 211.

[9] D. Ida, "Brane-world cosmology," JHEP 0009 (2000) 014 gr-qc/9912002.

[10] S. Parke, "Gravity and the decay .of the false vacuum," Phys. Lett. 121B (1983) 313.

[11] S. C. Davis, W. B. Perkins, A. C. Davis and I. R. Vernon, "Cosmological phase transitions in a brane world," Phys. Rev. D 63 (2001) 083518 hep-ph/0012223.

[12] A. D. Linde, "Decay Of The False Vacuum At Finite Temperature," Nucl. Phys. B 216 (1983) 421 [Erratumibid. B 223 (1983) 544]. 